\title{
Diffuse Idiopathic Skeletal Hyperostosis
}

National Cancer Institute

\section{Source}

National Cancer Institute. Diffuse Idiopathic Skeletal Hyperostosis. NCI Thesaurus. Code C84671.

A rare degenerative disorder that affects the spine. It is characterized by ossification of ligaments resulting in calcifications along the sides of the spinal vertebrae. 